\title{
Del ayer, del hoy y del mañana de la antropología en Cataluña: en homenaje a Claudio Esteva Fabregat ${ }^{1}$
}

\author{
Luis Calvo Calvo \\ IMF-CSIC \\ lcalvo@imf.csic.es
}

Resumen: Este artículo transcribe una conferencia sobre el desarrollo de la antropología en Cataluña y su inserción social y pública desde 1900 hasta ahora, así como los desafíos de la disciplina en nuestro siglo.

Palabras clave: antropologia; Catalunya; presència pública; prospectiva; desafiaments.

Abstract: This article transcribes a lecture aiming to reflect on the development and social and public insertion of Anthropology in Catalonia from 1900 up to the present as well as the challenges of the discipline in the twenty-first century.

Keywords; anthropology; Catalonia; public presence; prospective; challenges.

1 Deseo mostrar mi más sincero agradecimiento a las siguientes personas por la revisión del texto y por las sugerencias que han enriquecido el artículo: Yolanda Aixelà, María Jesús Buxó, Araceli González, Carmen Ortiz, Joan Prat y Ricardo Sánchez. 


\section{Proemio}

El profesor Claudio Esteva Fabregat falleció en Barcelona el 4 de septiembre de 2017 a la edad de 98 años, ${ }^{2}$ a escasos dos meses de que se cumpliesen cuarenta años del I Congreso Español de Antropología que él organizó y presidió en Barcelona en 1977.

Al cabo de unos días de su fallecimiento, L'ITA. Associació d’Antropologia organizó sus primeras Jornades d'Etnologia del Camp de Tarragona (Arxiu Municipal de Reus, 16 de septiembre de 2017). El presidente del ITA, el profesor Agustí Andreu, junto con el profesor Joan Josep Pujadas, tuvo la amabilidad de invitarme a impartir la ponencia que abría dichas jornadas: quiero dejar constancia de mi agradecimiento por esta invitación y por permitirme presentar mis ideas sobre la situación actual de la antropología en Cataluña y, en alguna medida, en España: de dónde venimos, dónde estamos, hacia dónde vamos y, sobre todo, cuáles son los retos para la disciplina en este complejo siglo xxi. Una vez finalizada mi presentación («L’Etnologia a Catalunya, passat i present. Línies de recerca emergents i perspectives de futur»), la directora de la revista Arxiu d'Etnografia de Catalunya (AEC), la profesora Yolanda Bodoque, tuvo la cortesía de proponerme que vertiese la presentación oral en un texto para ser publicado en el AEC. Amén de agradecer el ofrecimiento, indiqué a la dra. Bodoque que una cosa es una conferencia y otra, un escrito, pero en recuerdo y reconocimiento al dr. Esteva Fabregat, tengo la osadía de reunir mis reflexiones sobre el ayer, el hoy y el mañana de la disciplina en Cataluña. Por ello, las posibles lagunas que puedan aparecer en el texto se deben en su mayor parte a este intento de verter por escrito la presentación oral de las jornadas, por lo que diversos datos se ofrecen aquí tan solo de manera sintética.

Posiblemente la razón última que me anima a escribir estas líneas es manifestar mi más sincero agradecimiento al dr. Esteva Fabregat por su magisterio: fue uno de mis maestros, hasta el punto que mi tesina estuvo dedicada a una parte concreta de su obra ("La Antropología Aplicada en la obra de Claudio Esteva Fabregat", Universidad de Barcelona, 1985), y asimismo tuvo la generosidad de dirigir mi tesis doctoral ("La Antropología en Cataluña", Universidad de Barcelona, 1989). Además, gracias a su persona, que por entonces dirigía el

2 Por desgracia, el dr. Esteva Fabregat no pudo recoger en persona su nombramiento como doctor honoris causa por la Universitat Rovira i Virgili, acto que tuvo lugar el día 10 de noviembre de 2017. 
Centro de Etnología Peninsular e Hispanoamericano del CSIC, llegué a este en 1985, donde mi primera tarea fue asumir la edición de los dos últimos volúmenes de Ethnica. Revista de Antropología. Por todo ello, igracias, don Claudio!

\section{Introducción}

El motivo central de mi conferencia fue mi preocupación por la relevancia social más allá del ámbito estrictamente académico de la antropología en Cataluña, y por ende, en España y en Europa ${ }^{3}$ Este aspecto ya fue señalado por J. J. Pujadas cuando en 2005 escribió un texto con motivo de los 25 años de la revista Quaderns ${ }^{4}$ del Institut Català d'Antropologia (ICA): sus reflexiones son de plena actualidad y las comparto totalmente por su acertado análisis de la realidad institucional de la antropología en nuestra sociedad, de manera especial, cuando se piensa en las nuevas generaciones.

Tal como decía, el motivo de mi intervención fue poner de manifiesto mi preocupación por la presencia social del discurso disciplinar más allá de los recorridos biográficos de unos u otros profesionales. Dos primeros ejemplos ilustran mi inquietud. La revista dominical de La Vanguardia fechada el 25 de junio de 2017 dedicó su reportaje principal al tema titulado "Qué hemos aprendido de la crisis". El texto, firmado por Félix Badia, se articulaba a partir de catorce entrevistas a personas de distintas profesiones que, en resumen, eran: tres sociólogos, dos periodistas y analistas económicos, un economista, un historiador de la economía, dos escritores, un experto en nuevas tecnologías, un psicólogo, un analista electoral, un profesor de marketing, y un antropólogo e ingeniero que, sin pertenecer a lo que coloquialmente se denomina como la academia, aplicaba sus conocimientos a otras materias — en este caso, el medio ambiente- Esta circunstancia puede ser vista desde el punto de vista de que la disciplina está consiguiendo su reconocimiento en nuestra sociedad mas, por otro lado y a diferencia de otras materias, todavía le resta camino para ampliar su proyección, relevancia e incidencia social.

\footnotetext{
3 En este sentido y mientras se redactaba el presente texto, se produjo el fallecimiento de la profesora Françoise Héritier en París, la noche del 14 y 15 de noviembre de 2017. El rotativo Le Monde publicaba esta triste noticia en portada en su edición del día 17 de noviembre, circunstancia que, en otras latitudes, es difícil de pensar que pueda ocurrir.

4 Vid. 06/2005b. Número Especial del 25. aniversario de la revista Quaderns de l'ICA, <http://www. antropologia.cat/antiga/quaderns-e/re_num_1/pujadas.htm $>$.
} 
La publicación de este reportaje coincidió en el tiempo con la aparición de un informe de la Comisión Europea (Dirección General de Investigación e Innovación $)^{5}$ titulado Integration of social Sciences and Humanities in Horizon 2020: participants, budgets and disciplines. 2 nd Monitoring report on SSH-flagged projects funded in 2015 under the Societal Challenges and Industrial Leadership priorities (eds. Bogdan Iustin Birnbaum, Philippe Keraudren, Tobias Strom y Theodoros Vavikis, 2017), en el que se analizan la presencia y la participación de las ciencias humanas y sociales en los calls y proyectos concedidos en el programa marco en curso, conocido comúnmente como H2020. En este informe, amplio en datos, se puede leer (p. 12):

Regarding the variety of SSH [Social Sciences and Humanities] disciplines in the funded projects, contributions from the fields of economics (26\%), political science and public administration (17\%) are well integrated while a few other SSH disciplines are underrepresented. This is especially the case for the human geography/demography and anthropology/ethnology, which contribute with only $3 \%$ of researches in funded projects with an SSH dimension [... A As in 2014, we observe that the Humanities remain underrepresented.

A pesar de que esta cita no es nada alentadora - más adelante se analizará con más profundidad este estudio-, hay que decir que, más allá de la situación descrita a nivel europeo, hoy, después de más de cinco décadas de institucionalización en España, el discurso y los planteamientos antropológicos han ido ganando terreno en la sociedad y ello ha significado que en un gran número de instituciones, especialmente públicas, se hayan creado órganos que incorporan el discurso antropológico. ${ }^{6}$

5 European Commission. Directorate General for Research and Innovation. Directorate B - Open Innovation and Open Science. Unit B.6 - Open and inclusive Societies.

6 Por ejemplo, en el Ayuntamiento de Barcelona existe una Comisionada de Inmigración, Interculturalidad y Diversidad, que dirige una antropóloga (Lola López Fernández). 


\section{Del ayer..., la antropología antes de la década de 1970}

En términos generales, se puede decir que las claves que marcaron una buena parte del recorrido antropológico antes de la llegada de Claudio Esteva Fabregat a Barcelona en 1968 fueron las siguientes:

1. Entender la cultura bajo el prisma de la biología, lo que hizo que, en buena medida, se pudiese hablar de la «naturaleza biológica de la cultura», lo que comportaba que «raza»y «etnia» se usasen de manera indistinta. Esta concepción de la cultura estaba íntimamente relacionada con otros elementos provenientes de diversas dicotomías surgidas a partir de recepción de las ideas darwinistas: razón vs. fe, tradición vs. modernidad, campo vs. ciudad, agricultura vs. industria, trabajo manual vs. trabajo mecanizado.

2. Dicha concepción estaba ligada íntimamente a una interpretación historicista y arqueológica de la etnología.

3. Los anteriores aspectos propiciaron que, además de la búsqueda de lo que se podría denominar como ideal type, se quisiese indagar en el conocimiento de la "mentalidad primitiva" y, por ende, "popular", como muestra de los estadios del pasado que habían llegado (y/o "resistido") a la "modernidad".

4. La compilación del folklore, en este caso catalán, tuvo como objetivo compendiar algunos de los aspectos señalados, siendo el estudio de las «costumbres populares» una de las máximas expresiones por y para mantener viva la "tradición» así como demostrar las supervivencias en la sociedad catalana, por ej., de la «mentalidad primigenia".

Así, en la siguiente referencia sobre los pastores, como máxima expresión de todo lo antedicho, se condensa esta preocupación por indagar en esa mentalidad como expresión de estadios anteriores de la humanidad que habían sobrevivido hasta el presente:

Un dels oficis que durant més segles ha resistit els embats dels corrents renovadors i que més refractari ha estat a les innovacions és el de pastor. Ha travessat capes i més capes de civilització i sempre s'ha mantingut en un ingenu i encisador primitivisme. El seu estudi permet copsar múltiples plecs de l’ànima humana i penetrar algunes vibracions anímiques en el seu pur primitivisme. Ens atrevim a creure que el pastor és l'ofici i estament social més interessant d'estudi, considerat sota el punt de vista etnogràfic, i més avui que abans, ja 
que no podent-se sostreure al vent del modernisme que trasbalsa la Humanitat i perd per moments el seu encís i color per a confondrés amb la buida grisor imperant. ${ }^{7}$

Asimismo, las siguientes palabras de Pere Bosch i Gimpera en el prólogo de una obra escasamente valorada en nuestra historia disciplinar como Las Razas Humanas (Barcelona: Instituto Gallach, 1927, vol. I, p. 2) son un buen complemento de estas ideas:

Cuál ha sido el origen de la humanidad; cómo ha comenzado su vida y su civilización; qué razas y variedades de pueblos la integran formando una gama infinta de matices que se traducen en distintos aspectos de su cultura material y espiritual, son otros tantos problemas previos para todo estudio histórico y solo partiendo de ellos es posible asentar sobre ellos una base firme el conocimiento de los epiosodios del gran drama de la historia. Al mismo tiempo, al plantearnos el problema del hombre y de sus razas, aprendemos a comprender mejor una cantidad extraordinaria de fenómenos de la historia y aún de la psicología de los pueblos modernos, que no dependen del desarrollo histórico como tal, y que proceden de los bajos fondos raciales o de supervivencias de sus estados primitivos de cultura o de su mentalidad originaria. Por todo ello, al ensancharse el horizonte de las ciencias históricas durante el siglo pasado, descubriéndose y estudiándose los distintos tipos de humanidad que pueblan las regiones más apartadas del globo y menos afectadas por las grandes civilizaciones históricas, a la vez que reconocían los restos de culturas emparentadas con aquellas en nuestro propio suelo mediante las investigaciones prehistóricas, o el folklore revelaba curiosas supervivencias primitivas en la mentalidad popular de los países de alta civilización [...].

Una de las características de la antropología anterior a la década de 1930 fue el hecho de que, junto con insignes estudiosos como los citados Carreras i Artau o Bosch i Gimpera, el mundo paraprofesional tuvo un papel de primer orden en la recopilación de algunas de las expresiones populares (canciones, refranes, leyendas, formas de habitabilidad, etc.) como manifestación de las tradiciones propias. Tal como han puesto de manifiesto diversos estudiosos, entre los que destaca Llorenç Prats, cabría analizar mucho más a fondo todo

7 Joan Amades i Gelats. «Resenya de l'obra de S. Vilarrasa La vida dels pastors». Butlletí del Centre Excursionista de Catalunya, vol. XLVI (Barcelona, 1936), pp. 85-86. 
lo que subyace bajo el apelativo de "tradición". En este sentido, Joan Amades representa el ejemplo por antonomasia de este tipo de labor recolectora, la cual ha sido muy criticada por aspectos como su escasa sistemática, la ausencia de referencias, etc., pero a la que también cabe reconocer y dotar de valor en su justa medida por su denodado esfuerzo recolector.

A pesar del esperanzador panorama que se dibujaba hacia los años veinte del siglo pasado, diversos factores hicieron que la disciplina como tal no consiguiese su consolidación en el ámbito académico antes de la Guerra Civil española, lo que hizo que el discurso antropológico se agotase en sí mismo. En Cataluña, algunas de las razones hacen referencia al papel destacado de la arqueología, la baja actividad del Arxiu d'Etnografia i Folklore de Catalunya a partir de la salida de Josep Maria Batista i Roca, así como la imposibilidad de llevar adelante grandes iniciativas como la del Museu d'Etnografia de Catalunya. Cabe decir que, a pesar de que la etnología no llegó a consolidarse en la universidad, actores como Amades siguieron trabajando de manera denodada e, incluso, ese momento se vio cómo una nueva manera de hacer etnografía empezaba a surgir de la mano de Ramon Violant i Simorra.

Después de la Guerra Civil y hasta la llegada de Claudio Esteva Fabregat a Barcelona a finales de la década de 1960, la disciplina continuó en el medio académico bajo el peso de la arqueología y de la Prehistoria: posiblemente, el principal motor disciplinar fue la creación, gracias a las responsabilidades institucionales de Carreras i Artau en el Ayuntamiento de Barcelona, del Museo de Industrias y Artes Populares (MIAP) (1944) y del Museo Etnológico de Barcelona (MEB) (1949), los cuales, en buena medida, fueron la plasmación de los ideales antropológicos existentes antes de la Guerra Civil. Ambos museos propiciaron que tanto Amades como Violant i Simorra tuviesen un ámbito institucional en el que llevar a cabo sus proyectos: de hecho, en las décadas de 1940 y 1950 se publicaron algunas de las grandes obras de Amades (entre otros, Costumari Catalá, Folklore de Catalunya, etc.) y los estudios etnográficos de referencia de Violant i Simorra (por ej., El Pirineo Español, Etnografía de Reus i la seva comarca, etc.).

La producción editorial de estos dos autores tuvo su contrapunto en el número significativo de obras que en las décadas de 1950 y 1960 vieron la luz bajo el epíteto de "las razas humanas". Junto con las publicaciones de carácter folklórico (Amades) y etnográfico (Violant), este tipo de obras tuvo una notable 
acogida popular y — básicamente - surgió de la pluma de August Panyella (en aquel momento, director del MEB y, a partir de principios de los años sesenta, también del MIAP). Este aspecto es, desde mi punto de vista, importante por el impacto social de tales libros que mantenían vivas las ideas antropológicas anteriores a la Guerra Civil. Así, en un manual de divulgación del momento, debido a un prehistoriador (Francisco Jordá Cerdá, Resumen de Etnología, Barcelona: Seix Barral, 1951, pp. 82-83), se puede leer:

La cultura moderna. La nueva cultura dotada de un movimiento expansivo se extendió por todo el mundo mediante la creación de los imperios coloniales que posibilitaron la adquisición de productos para la alimentación de la nueva economía industrial [...] En todos los órdenes se van imponiendo en el mundo entero sus elementos culturales y todos los renacimientos nacionalistas de los países no europeos se hacen siempre tomando como base los fermentos activos de la moderna cultura occidental. Ello es buena prueba de su vitalidad y de su eficacia como fórmula cultural todavía activa.

Habría que situar este texto en el contexto de aquel momento, pero desde mi punto de vista, lo relevante es que en nuestra sociedad actual la concepción de las culturas humanas siga anclada, en muchos casos, en aquel ideario. Así, y en referencia al Museu de les Cultures del Món, inaugurado en 2015 con una gran inversión económica, en la web Tripadvisor.es se pueden leer comentarios como los siguientes (las cursivas son mías):

Una vuelta al mundo por mil culturas [opinión escrita el 21 de abril de 2016]. En pleno centro del Barrio Gótico. En un edificio histórico perfectamente remodelado. Un paseo por culturas exóticas, muchas desconocidas, especialmente de Oriente. Con salas llenas de objetos muy bien expuestos. Costumbres, vestimenta, tradiciones, enormes piezas de madera labrada, joyería, artes, cerámica. Un sinfín de razones.

Oasis de paz en el Born [opinión escrita el 26 de marzo de 2017]. La colección no es extensa y está muy bien presentada, así que resulta un gusto pasear tranquilamente sin prisas e ir descubriendo artefactos de lugares remotos. Muy recomendable.

Comentarios como los anteriores deberían llamar a la reflexión sobre el impacto social del discurso antropológico y, de manera especial, sobre la ca- 
pacidad de intervención y de presencia pública de la antropología, mucho más porque, después de un siglo, todavía no se ha conseguido que la etnología en Cataluña haya podido crear un museo propio, como sí lo han hecho otras disciplinas como la arqueología o la historia. Las razones pueden parecer evidentes, mas cabe recordar que Carreras Artau y Telesforo de Aranzadi ya plantearon la necesidad de crear un Museo de Etnografía y Folklore de Cataluña en una conferencia en el Centre Excursionista de Catalunya el 28 de noviembre de 1916, y dejando de lado la creación del MIAP y del MEB, la Generalitat de Catalunya organizó un seminario para la creación del Museu Nacional d'Etnologia de Catalunya los días 28 y 29 de marzo de $1993,{ }^{8}$ asunto que periódicamente surge de nuevo. ${ }^{9}$ Tal como apuntaba antes, cabría reflexionar sobre las razones por las que una disciplina como la antropología no ha conseguido aunar esfuerzos para hacer factible un proyecto que, más allá de las lógicas diferencias en cuanto a su concepción y diseño, podía haber sido un dinamizador de la disciplina en el entorno social, tal como en otras latitudes se ha logrado.

8 Vid. Silvia Ventosa."Seminari sobre el futur Museu Nacional d'Etnologia de Catalunya”. Revista d'Etnologia de Catalunya, 5 (Barcelona, julio de 1994), p. 162.

9 Vid."Els museus d'etnologia i societat a debat",Barcelona, CaixaForum, 2, 3 y 4 de febrero de 2005. Cf."Els museus d'etnologia i societat a debat. Presentació". Nadja Monnet i Xavier Roigé [quaderns-e 09/2007a] del Institut Català d'Antropologia; Xavier Theros. "Exòtics i familiars. La nova xarxa de Museus d'Etnologia obre el debat sobre el futur d'aquestes institucions". El Pais, Quadern de Catalunya (1 de mayo de 2008); Generalitat de Catalunya [documento de trabajo]. Museu Nacional d'Història, Arqueologia $i$ Entologia de Catalunya. 26 de marzo de 2009) 


\section{Institucionalización y consolidación}

Como es bien conocido, Esteva Fabregat llegó a Barcelona en 1968 para incorporarse a la Universidad de Barcelona como profesor agregado de Etnología así como para asumir la dirección - iy refundación! - del Centro de Etnología Peninsular del CSIC que había sido creado en 1948 por Agustí Duran i Sanpere y Julio Caro Baroja en Barcelona y Madrid, respectivamente. Cabe decir que, desde su fundación, este centro había tenido una trayectoria poco significativa a pesar de las expectativas con que se creó.

Esteva Fabregat consiguió que se crease la primera cátedra de Antropología Cultural en España en la UB en 1971 y, a raíz de ello, el primer departamento de la disciplina en España en 1972, hechos que, vistos con la perspectiva del tiempo, fueron realmente notables en el panorama universitario español del momento por lo que suponían de renovación conceptual y metodológica, ya que, por ejemplo, en aquellos años publicar obras como Estado, etnicidad y biculturalismo (Barcelona: Península, 1984) o Antropología industrial (Barcelona: Anthropos, 1984) era una absoluta novedad, así como, por ejemplo, realizar trabajos de campo no tan solo en la Península Ibérica sino también en América con fuerte acento interdisciplinar.

Dichas novedades se aprecian mejor teniendo en cuenta el horizonte teórico, metodológico e institucional de la antropología española al inicio de la década de 1970, marcada por aspectos como la dependencia de la Prehistoria y la Arqueología (cabe recordar que las plazas que salían a concurso solían tener la denominación de "Prehistoria y Etnología"), el uso de métodos descriptivos, el autodidactismo y la baja profesionalización, la ausencia de financiación, tanto en lo referido a recursos humanos como materiales, o la falta de planes de estudio. ${ }^{10}$

Esta renovación se valora mucho más si se lleva a cabo un mínimo ejercicio comparativo con lo que aquí era la antropología y la situación de la disciplina a nivel internacional. Así, cabe recordar que en aquel tiempo (1969) la prestigiosa publicación Current Anthropology se interesaba por las responsabilidades sociales de la antropología y Les Temps Modernes (1970-1971) dedicaba dos volúmenes al tema de «Anthropologie et colonialisme». Por su parte, en Bar-

10 Claudio Esteva Fabregat. La Etnología española y sus problemas. Zaragoza: Institución Fernando el Católico, 1969. Citado por Joan Prat. Antropología y Etnología. Madrid: Editorial Complutense y Caja de Ahorros y Monte de Piedad de Madrid, 1992, p. 21. 
celona se inauguraba en 1973 la nueva sede del Museo Etnológico de Barcelona gracias al denodado esfuerzo de August Panyella (cabe mencionar que esta sede fue el primer museo de nueva planta construido hasta aquel momento en Barcelona en el siglo $\mathrm{xx}$ ). Este museo era en aquellos instantes la institución dedicada a la disciplina que contaba con mayores recursos y su planteamiento conceptual y metodológico respondía, en lo básico, al modelo heredado de Bosch i Gimpera y Carreras i Artau tal como, por ejemplo, se puede apreciar cuando Panyella presentó al Ayuntamiento de Barcelona el proyecto para realizar una expedición a Etiopía que, finalmente, se llevó a cabo en los meses de enero y febrero de 1974. En el informe preliminar para la aprobación de dicha expedición y la consiguiente dotación de recursos económicos, se puede leer:

[...] una expedición etnológico-antropológica a Etiopía, dado el extraordinario interés raciológico, cultural y religioso de este país [...] a) Interés excepcional de la zona del macizo de Abisinia como centro de la dispersión de la raza etiópica, núcleo raciológico de transición entre európidos y melánidos. b) Zona de confluencia de lenguas y culturas de origen cuchita (camita) y semita. c) Confluencia de religiones precristianas, cristianismo, judaísmo y mahometismo. d) Gran tradición político-cultural y artesanía (Fondo documental MEB).

Asimismo, Panyella anotaba en su cuaderno de viaje lo siguiente (aeropuerto de Roma, 17 de enero de 1974, en tránsito para Adis Abeba):

Viaja un interesante subgrupo — creo filipinos - antropológicamente «polimorfos»: 1 paleo mongol, una proto-malaya, de raíz de nariz hundida, mentón huidizo, labios gruesos, 1 al parecer híbrido de tagalo + español, 1 levemente negroide de piel —acaso igorrote - de rasgos algo primitivos (Fondo Documental MEB).

Frente a esta visión, Esteva planteó nuevas dimensiones del trabajo antropológico con la introducción de conceptos como "proceso", "cambio social", "aculturación” o "procesos de urbanización”, y todo ello desde "[... ] la aplicación del enfoque etnográfico, es decir, la observación directa y personal de los fenómenos que son objeto de estudio", ${ }^{11}$ enfoque que comportaba una investigación "[...] dinámica y empírica, ya que la teoría sigue a los datos. Es tam11 Joan Frigolé. “Crónicas”. Éthnica 1 (Barcelona, 1971), p. 232. 
bién experimental, ya que pondrá en evidencia la clase de condicionamientos y determinismos relativos que concurren o han concurrido en el desarrollo de las diferencias culturales [... ]" ${ }^{12}$ Esta concepción fue aplicada por Esteva y sus primeros discípulos en investigaciones que tenían por objeto, por ejemplo, "[... ] obtener materiales sobre aculturación y cambio social en el Alto Aragón. El desenvolvimiento teórico [... ] consiste en verificar la tesis de que el paso de la economía familiar ganadera al empleo asalariado fijo ha constituido el punto de partida para la urbanización cultural de esta región",13 y que conllevaban trabajos de campo que generaron, entre otras, publicaciones como "Para una teoría de la aculturación en el Alto Aragón" (Claudio Esteva Fabregat. Etbnica 2 [Barcelona, 1971], pp. 6-75) o "Elementos para un modelo del proceso urbanización/desruralización en el Alto Aragón” (Dolores Comas y Juan José Pujadas. Ethnica 9 [Barcelona, 1975], pp. 37-74).

El dinamismo que Esteva introdujo tuvo pronto sus resultados con la realización de un conjunto de tesinas que, por la variedad de temáticas y enfoques, se convirtieron de por sí en la prefiguración de algunos de los nuevos ámbitos y derroteros de la investigación antropológica en Cataluña en los años venideros. $^{14}$

El proceso de institucionalización propició la consolidación de la primera generación de antropólogos con formación universitaria específica, consiguiendo múltiples logros en muchos ámbitos, por ejemplo, en dotaciones de personal en los departamentos universitarios (en 2009 se contabilizaban 69 profesores y personal investigador en las universidades y en el CSIC en Cataluña) junto con la organización de congresos internacionales como la IV Conferencia de la EASA en Barcelona en 1996, participación en workshops, congresos y proyectos de investigación internacionales, publicaciones en editoriales internacionales de referencia, etc., que han configurado la antropología en Cataluña en estas

\section{Ibidem,}

13 Joan Frigolé. «Crónicas». Éthnica 2 (Barcelona, 1971), p. 228.

14 Entre otras, Josefina Roma: “Los A-ni'to y su función en la sociedad Igorrote de Filipinas”; Joan Frigolé; "Estudio histórico-sociológico de la vila de Bañolas en el año 1900"; Jesús Contreras: "Empirismo y superstición en la medicina popular y en la medicina académica del siglo xvirI"; Dolores Soriano: "Aspecto socioeconómico de un pueblo del Pirineo: Campellas"; María Jesús Buxó: "Etnografía del habla: modelos de relación diádica en Catalunya”; Montserrat Camps: "Tópicos y estructura social”; Joan Prat: "Estudio de los cuentos infantiles"; Manuel Moreno: "Essai sur le Don". 
últimas décadas. Cabe decir, no obstante, que, como todo proceso de institucionalización, no estuvo exento de dificultades, de todo tipo, que, pasado ya medio siglo, posiblemente debería ser objeto de análisis, sobre todo pensando que, en estos momentos, la generación que ha configurado la antropología en Cataluña en estas últimas cinco décadas ha llegado o está cercana a la jubilación. Frente a esta situación y más allá de unas u otras trayectorias personales, cabe preguntarse, por ejemplo, si el cambio generacional y la renovación universitaria están aportando diseños que permitan innovar en el plano teórico y en la actividad profesional, así como si existen propuestas que permitan que la antropología pueda jugar un papel social más relevante.

En relación a todo ello, un dato significativo que puede aportar elementos para la reflexión es el número de tesis doctorales leídas en Cataluña (básicamente UB, UAB y URV) desde principios de la década de 1980 hasta 2016: aunque el que suscribe ha comprobado que existen algunas deficiencias, la información que aporta la base de datos TDX [Tesis Doctorals en Xarxa] del Consorci de Serveis Universitaris de Catalunya (CSUC) es relevante. En el citado periodo se han leído 367 tesis doctorales de antropología que tuvieron como objeto de estudio los siguientes ámbitos espaciales: 180 Cataluña, 44 España y 143 extranjero. Estas cifras permiten ver que, globalmente, las tesis realizadas en el espacio ibérico superan las que han tenido como objeto de estudio otros ámbitos espaciales. Aún siendo positivos los datos en su conjunto, cabría preguntarse, por ejemplo, el porqué de la elección de uno u otro ámbito espacial o unas u otras temáticas. Posiblemente, una de las razones - esgrimida ya tradicionalmente - haya sido la infrafinanciación que ha impedido el desarrollo de proyectos de investigación de mayor alcance. A la vista de lo realizado, posiblemente cabría pensar en la necesidad de buscar mecanismos que permitan llevar a cabo nuevas actuaciones para articular y proyectar mejor la disciplina; en este sentido, iniciativas como el Máster Interuniversitario en Antropología Médica y Salud Global (URV/UB/CSIC) es un buen ejemplo de cómo aunando esfuerzos se pueden obtener destacables resultados.

Mas como ya se ha apuntado, el proceso de consolidación y desarrollo ha tenido sus deficiencias. Una de ellas hace referencia al peso institucional de la disciplina, el cual se puso de manifiesto, por ejemplo, con el destino final de los archivos (miles de horas de grabaciones, fotografías, cuadernos de campo) de Lluís Mallart. Por desgracia y después de múltiples gestiones, su ubicación ha 
sido la Biblioteca Éric-de-Dampierre de la Université de Paris Ouest, donde se pusieron todos los medios para la ordenación y digitalización de los materiales. ${ }^{15}$

Por tanto, partiendo de que el proceso de consolidación de la disciplina ha sido satisfactorio en muchos aspectos (así, cabe recordar cómo diversos antropólogos han asumido altas responsabilidades institucionales en la UB o han trabajado en las comisiones del diseño curricular para la implantación del Plan Bolonia), cuando se han realizado estudios sobre el estado de la disciplina se ha incidido en una serie de aspectos que no cabe olvidar, de manera especial si se tiene la ambición de definir nuevos escenarios institucionales, docentes e investigadores. Así, el Institut d'Estudis Catalans impulsó los denominados Reports de la Recerca, ${ }^{16}$ que abarcaron el periodo 1993-2009 y que se materializaron en tres estudios. En ellos, se pusieron de manifiesto algunas de las preocupaciones de los antropólogos catalanes - que podrían ser asumidas también por otras áreas de conocimiento-, siendo algunos de los aspectos más destacados los siguientes:

1. La desvinculación entre docencia e investigación se considera como uno de los más graves defectos de la antropología en Cataluña.

2. Programas de estudio clásicos que no son acompañados por otros instrumentos que capaciten profesionalmente a los estudiantes y les permitan hacer frente a las nuevas realidades y sus problemáticas asociadas.

3. Hasta el diploma de estudios avanzados (DEA) y los estudios de doctorado, no existe un «espacio» docente para el trabajo de campo ni tampoco dotaciones económicas que den soporte a la tarea de los departamentos para potenciar este aspecto.

4. Ausencia de una formación profesional más allá de los límites del propio país y del sistema docente.

5. Dificultad de captar estudiantes, de manera especial desde que la antropología es materia de segundo ciclo.

15 Después de ello, el material físico recaló en el Arxiu Nacional de Catalunya, aunque, tal como se comunicó al propio Lluís Mallart, a día de hoy no hay ni recursos humanos ni materiales para su estudio, ordenación, digitalización, etc. Gracias a las gestiones de la dra. Yolanda Aixelà, en la Institución Milá y Fontanals del CSIC (Barcelona) existe una copia del material digitalizado.

16 Vid. <https://www.iec.cat/reports/>. 
6. Dada la fragilidad institucional de la disciplina, posiblemente podría ser acertado crear departamentos de ámbito interuniversitario.

7. Las infraestructuras son, en términos generales, lamentables, y en muchos casos, la tónica es la ausencia de laboratorios, libros, despachos o ayudas para la realización de actividades académicas (seminarios), que se llevan a cabo gracias al voluntarismo de las personas.

8. La proyección pública ha aumentado en los últimos lustros pero parece que está más ligada a recorridos biográficos que a la consideración de la importancia de la disciplina como tal.

Como se puede apreciar a lo largo del presente texto, una de mis preocupaciones fundamentales es destacar el papel y el lugar institucional de la disciplina en el contexto universitario y científico; de esta manera, un buen ejercicio al respecto sería comparar cuál ha sido el recorrido institucional de nuestra disciplina con el de otra tan cercana a la antropología como es la psicología. Teniendo presentes los datos ya conocidos de la institucionalización de la antropología, cuando Esteva empieza a trabajar en la Facultad de Filosofía y Letras de la UB, en el curso 1969-1970, los "Estudios de Psicología” ya se inician en esta misma facultad; el 10 de enero de 1974 se aprueba el Plan de Estudios de Primer Ciclo en dicha facultad (Plan 1974), el 1 de octubre de 1977 se aprueba el Plan de Estudios de Segundo Ciclo, en 1983 se crea la Facutad de Psicología en la UB y, finalmente, en 1992 se constituye el Col.legi Oficial de Psicologia de Catalunya. En mi opinión, tales datos deberían llamar a la reflexión, de manera especial en lo referido a la profesionalización. Y, en este sentido y ampliando el marco al conjunto de la antropología española, cabría pensar en por qué en un momento determinado (V Congreso de Antropología, Granada, 10-14 de diciembre de 1990) se apostó por la creación de la Federación de Asociaciones de Antropología del Estado Español (FAAEE) — que se declaró como «el nexo de coordinación y comunicación múltiple entre las asociaciones de antropología del Estado español»- y no tanto por la constitución de un colegio profesional, aspecto que en Granada propusieron Teresa San Román, Aurora González y María Jesús Buxó. Después de cinco lustros, cabría preguntarse si ambas iniciativas eran excluyentes entre sí o, por el contrario, la unión de ambas hubiese dotado de mayor peso institucional a nuestra disciplina. 
A partir de la década de 1980, y de manera especial en la de los noventa, nuevos actores empezaron a contribuir a dinamizar la antropología en Cataluña: de una parte, las políticas de la Generalitat de Catalunya y, de otra, las asociaciones de carácter paraprofesional en las que, en muchos casos, se aunaban los intereses civiles con los profesionales alrededor de nuevos ejes de estudio, como fue el caso del concepto de "patrimonio etnológico". Así, entidades como la Associació Catalana de Patrimoni Etnològic (ACPE), ${ }^{17}$ Carrutxa (Reus), el Museu Etnològic del Montseny (Arbúcies), el Ecomuseu de les Valls d’Àneu (Esterri d’Àneu), el Museu de la Pesca (Palamós) o el Museu de les Terres de l'Ebre (Amposta) se convirtieron en grandes motores de la actividad etnológica, donde confluyeron intereses profesionales de antropólogos con los de estudiosos de carácter local de gran valía etnológica, como fue la colaboración entre la ACPE y el Consell Cultural de les Valls d’Àneu para llevar adelante el proyecto del Ecomuseu de les Valls d’Àneu. Por su parte, la Generalitat de Catalunya, desde un primer Centre de Documentació i Recerca de la Cultura Tradicional i Popular (CDRCTP), ${ }^{18}$ con posterioridad Centre de Promoció de la Cultura Popular i Tradicional Catalana, que surgió gracias a la ley 2/1993, de 5 de marzo, de fomento y protección de la cultura popular y tradicional y del asociacionismo cultural, por primera vez ofrecía un marco jurídico en todo lo referido al patrimonio etnológico. Con posterioridad, este marco legislativo se vio ampliado con la aprobación de la ley catalana de patrimonio cultural (ley 9/1993 de 30 de septiembre _DOGC núm. 1807, de 11 de octubre de 1993-). ${ }^{19}$

Cabe deternerse en la ley 2/1993, ya que, tal como se ha dicho, por primera vez abordaba el concepto de "patrimonio etnológico» y, lo que era más importante si cabe, el mandato de creación del Inventario del Patrimonio Etnológico de Catalunya (IPEC). Así, esta ley, en su artículo 5, definía aquel así: “[...] a. Els immobles i les instal.lacions emprats consuetudinàriament a Catalunya les característiques arquitectòniques dels quals siguin representatives de formes

17 Vid. Montserrat Iniesta. "L'Associació Catalana del Patrimoni Etnològic". Revista d'Etnologia de Catalunya 2 (Barcelona, febrero de 1993), pp. 170-172.

18 Vid. Antoni Anguela. "Centre de Documentació i Recerca de la Cultura Tradicional i Popular". Revista d'Etnologia de Catalunya, 1 (Barcelona, 1992), pp. 80-87.

19 Este marco legislativo se ha visto ampliado con el Acord de Govern de la Generalitat de Catalunya de 23 de enero de 2017 por el que se aprueba el anteproyecto de ley del patrimonio cultural inmaterial catalán y del asociacionismo cultural. 
tradicionals. b. Els béns mobles que constitueixen una manifestació de les tradicions culturals catalanes o d'activitats sòcio-econòmiques tradicionals. $c$. Les activitats, els coneixements i els altres elements immaterials que són expressió de tècniques, oficis o formes de vida tradicionals", e instó a la elaboración del IPEC ("El Govern ha d'elaborar l'Inventari del Patrimoni Etnològic de Catalunya, en el qual s'han de recollir tots els béns integrants d'aquest patrimoni”). El hecho de que el marco legislativo no prefijase de antemano cómo se debía realizar el IPEC ni tampoco redujese el ámbito temático, permitió diseñar una estrategia novedosa que propició que se configurase como gran instrumento para el fomento de las investigaciones antropológicas en Cataluña en general, ya que, conceptual y metodológicamente, se diseñó desde aquellas perspectivas que a partir de la década de 1970 configaron la antropología en Cataluña: "cambio", "proceso"... Por otra parte, la posibilidad de contar con nuevos recursos económicos (en el periodo 2003-2009 se destinaron 1.296.000€) permitió la realización de numerosos estudios con alto valor añadido.

Merece especial mención cómo la Generalitat de Catalunya se implicó en la promoción y financiación de un concepto tan novedoso en aquel momento como el de "patrimonio etnológico". Sin olvidar las implicaciones políticas del fomento de un concepto como este - desarrollado fundamentalmente a partir de los trabajos de la Mission du patrimoine ethnologique francesa- puede afirmarse que la coincidencia de intereses de los profesionales, ya fuesen académicos o estudiosos de asociaciones o museos como los citados con anterioridad, y de la Administración permitió dar un giro significativo que propició que, desde 1993, el IPEC se convirtiera en uno de los motores del trabajo etnológico en Cataluña. Y, en ese sentido, hay que mencionar la figura de Antoni Anguela i Dotres (1947-2010), ${ }^{20}$ responsable del CDRCTPC y después del Àrea de Patrimoni Etnològic del CPCPTC: sin su generosidad personal y su decidido apoyo institucional difícilmente se hubieran llevado a cabo actuaciones como la exposición "El món de Joan Amades" (1990), un primer gran revulsivo que sentó las bases de futuras actuaciones como la creación por el CDRCPTC de la Revista d'Etnologia de Catalunya, jornadas de análisis como Ethnographica ( Terrassa, diciembre de 1993), los debates con la ACPE (II Jornades de la ACPE, Esterri d’Àneu, 29 de octubre-1 de noviembre de 1993) o la exposición «De

20 Vid. Lluís Calvo. "Antoni Anguela i Dotres. La passió per la cultura popular i tradicional i el patrimoni etnològic de Catalunya". Revista d'Etnologia de Catalunya, 37 (Barcelona, diciembre de 2010), pp. 192-194. 
l'ahir i de l'avui. El patrimoni etnològic de Catalunya» (Barcelona, Centre de Cultura Contemporània de Barcelona, noviembre de 1995-febrero de 1996), que, en definitiva, crearon un clima de confianza institucional que permitió llevar adelante el IPEC, ${ }^{21}$ así como futuras actuaciones, en la actualidad, como el Observatori o les Jornades Nacionals de Patrimoni Etnològic, y colecciones de publicaciones como Temes d'Etnologia, dirigida exitosamente durante años por Joan Prat i Caròs. En estos momentos, a los 25 años de la creación del IPEC, posiblemente fuese adecuada una reflexión sobre el propio concepto de "patrimonio etnológico" — pienso, sinceramente, en si los archivos de Lluís Mallart no deberían tener cobijo en el propio IPEC si se tiene un concepto amplio del término - o si habría que buscar otros instrumentos que permitiesen profundizar en la importancia del IPEC como, por ejemplo, convocatorias de proyectos marco dirigidos a estudiar temas concretos que afectan a todo el territorio, tales como el agua, hábitat...

\section{Una antropología para un nuevo milenio}

Cualquier ejercicio que pretenda apuntar posibles direcciones o tendencias de trabajo en los momentos actuales puede ser tildado, cuando menos, de pretencioso y atrevido. A pesar de ello, me aventuro a pergeñar unas sucintas ideas que doten de base conceptual a los planteamientos que se exponen más adelante. ${ }^{22}$

1. Reflexividad antropológica. Como es conocido, la investigación científica, pero de manera especial la antropología, se caracteriza por una continua revisión, crítica, de sus planteamientos; de esta manera, la «reflexividad antropológica» supone, entre otras cosas, una nueva manera de escribir en la disciplina, un nuevo estatus para la descripción y la interpretación, así como el análisis de sus implicaciones. Asimismo, comporta la implicación, en primera persona, del antropólogo en la construcción de modelos sociales y culturales y también se plantea la reflexión dialógica sobre cómo los sujetos objeto de estudio reflejan sus propias vidas y construyen su universo de prácticas y creencias. Según

21 Manel Català, Roger Costra i Rafel Folch. “Balanç de catorze anys de l'Inventari del Patrimoni Etnològic de Catalunya". Revista d'Etnologia de Catalunya, 33 (Barcelona, noviembre de 2008), pp. 118-141.

22 Vid. Mondher Kilani. Anthroplogie. Du local au global. Paris: Armand Colin, 2012, pp. 336-345. 
esto, los sujetos tienen la capacidad de dialogar sobre los modelos que les describen y hablar —o adquirir incluso - un protagonismo en la coautoría de las obras. Por ello, el antropólogo se ve inmerso en una mayor complejidad de las versiones sobre las posibilidades reales de describir las diversidades.

2. Crítica del concepto de "sociedad". Las categorías tradicionales de lo que venía en denominarse "organismo social" (escuela, familia, hospital, ejército, empresa, iglesia, etc.) se convierten en más fluidas, dada la creciente interrelación entre acontecimientos locales y globales.

3. Segunda Modernidad. Por todo lo antedicho - y siguiendo las ideas de Z. Bauman-, aquel "organismo", con sus bien definidas categorías, se ve enfrentado a nuevas situaciones, por lo que se puede hablar de "sociedades sólidas vs. fluidas", y, así, categorías tradicionales ligadas a la "estabilidad del organismo" cada vez se presentan como más obsoletas. De esta forma, "fuerza","cuerpo social", "infraestructura" y "superestructura" decaen frente a otras como "conexión", "fluidez" y, sobre todo, "red”, "contingencia", "movilidad" e "imprevisibilidad".

4. Disolución de los entornos sociales tradicionales. El conjunto de aspectos hasta ahora descritos surge, tal como ha comentado A. Appadurai en diversas obras, del aumento constante de los flujos económicos, culturales y migratorios desarrollados a partir de una creciente y mayor interpenetración cultural, cuya expresión popular toma el nombre de "mundialización" o "globalización".

Estas variables tienen como traducción, y en palabras de M. Godelier, ${ }^{23}$ que la antropología debe hacer frente a un doble movimiento como es una mayor y creciente integración de las economías junto con una reafirmación de las identidades locales o nacionales. Mas este conjunto de aspectos contiene, en sí mismo, un peligro para la antropología: la provincialización — para algunos «aldeanización»-, y por ende, su marginalidad en caso de que no conecte con las tendencias y dinámicas globales, algunas de las cuales se han esbozado brevemente antes. Ese "peligro" viene sobre todo por el aumento, significativo, de las microetnografías, y a partir de ahí, que la disciplina solamente sea conside-

23 Maurice Godelier. Communauté, Société, Culture. Trois clefs pour comprendre les identités en conflits. Paris: CNRS Éditions, 2009, p. 61. 
rada como una «descriptora de experiencias». Si esta tendencia se impone, posiblemente la antropología pierda buena parte de su sentido final. Tal como ha escrito C. Geertz, ${ }^{24}$ la antropología debe pasar de las "miniaturas etnográficas" a los "frescos murales", lo que recuerda que no debe perder nunca la "desterritorialización geográfica e ideológica" que contribuya a la búsqueda de explicaciones universalistas sobre el hecho cultural. ${ }^{25}$

Pero más allá de estas reflexiones que afectan estrictamente a la propia idiosincrasia del pensamiento antropológico, cabe pensar en qué antropología debe llevarse a cabo en este siglo xxi. Tal como indica el título de este apartado, el reto de la disciplina está en plantearse qué discurso y qué actuación tiene que llevar a cabo. Si su papel tradicional ha venido definido por el de "participaciónobservación", diversos autores ya están hablando de que este binomio debe pasar a ser el de "participación observante", postura que plantea que el antropólogo debe romper la distancia con los sujetos de estudio con el fin de posibilitar la restitución de los conocimientos de las comunidades objeto de estudio, y en la medida de lo posible, que aquellos se conviertan en instrumentos de mejora colectiva. En este sentido, la tan famosa «antropología aplicada», que Esteva Fabregat ${ }^{26}$ defendió durante mucho tiempo, ¿no debería ser, realmente, uno de los ejes que marquen el devenir de nuestra antropología en este siglo xxI?

Tal planteamiento surge, de manera especial, cuando se piensa en el papel de la disciplina frente a lo que viene en denominarse como «tendencias y dinámicas globales» que podrían resumirse en los siguientes grandes ejes: 1 . Más allá de Shangái: el tiempo de la urbanización; 2. La punta del iceberg: acelerar el cambio tecnológico; 3. Hacerse viejo no es lo que solía ser: responder a los desafíos de un mundo que envejece; 4. Comercio, personas, finanzas y datos: mayores conexiones globales (Richard Dobbs, James Manyika y Jonathan Woetzel. The four global forces breaking all the trends. Chicago: McKinsey Global Institute, 2015. Vid. <https://www.mckinsey.com/business-functions/strategy-and-corporate-finance/our-insights/the-four-global-forces-breakingall-the-trends $>$ ). Estos aspectos deben, necesariamente, tener en cuenta otros

24 Vid. supra Kilani, p. 343.

25 Ibidem, p. 340.

26 Vid. "La Antropología Aplicada y su problemática". En: Alfredo Jiménez (coord.). Primera reunión de antropólogos españoles. Sevilla: Universidad de Sevilla, 1978, pp. 253-321. 
factores de primer orden como son los referidos al cambio global e, incluso, a la violencia y a la guerra.

Si volvemos al informe Integration of Social Sciences and Humanities in Horizon 2020: participants, budgets and disciplines de la Unión Europea sobre el papel de las ciencias humanas y sociales en el vigente programa marco (H2020) (vid. supra nota 5), los datos referidos a la disciplina son poco alentadores. Tal como ya se indicó con anterioridad, si se analiza en profundidad este documento, la participación de antropólogos en las convocatorias del H2020 en curso suma tan solo un 4\%. Cabe tener presente que el presupuesto inicial de los calls en 2105 fue (descontando el referido SC6) de 3.618M€. Siendo preocupantes estos datos, mucho más lo son cuando se analiza la participación de las ciencias sociales y de las humanidades en los diferentes programas. Así, por ejemplo, y en el caso de la antropología, su participación en los grandes programas del H2020 es la siguiente: Salud (SC1), 1\%; Alimentación (SC2), 1\%; Energía (SC3), 1\%; Transporte (SC4), 0\%; Clima (SC5), 1\%; Sociedades inclusivas (SC6), 4\%; Seguridad (SC7), 0\%. Este conjunto de datos llama, cuando menos, a la reflexión, ya que, desde mi punto de vista, esta escasa participación propicia que se corra el riesgo ya no solo de no participar en los proyectos de investigación propiamente dichos, con todo lo que ello conlleva (financiación, contrataciones, etc.), sino, sobre todo, que el discurso antropológico sea visto como residual, sin capacidad de participación e intervención en los retos y desafíos que la UE se ha fijado para este periodo. Estar escasamente presente o no participar en los programas que afectan muy directamente a la situación de la población europea comporta un gravísimo riesgo para el futuro de la disciplina, sobre todo porque es difícil de entender que la antropología no tenga nada que decir en ámbitos como la alimentación, el transporte, el clima o la seguridad.

La Comisión Europea es consciente de que "[... ] many scientists are still reluctant to engage into inter-disciplinary research (even amongst $\mathrm{SSH}$ disciplines) because of complex inter-knowledge issues, but also because of the social constraints by disciplines, specialization and careers" (p. 65). Por esta misma razón, pero sobre todo a los 40 años del I Congreso Español de Antropología, posiblemente, fuese este un buen momento para plantearse si aquella perspectiva disciplinar integradora que Esteva Fabregat postulaba y defendía no debería ser al menos objeto de reflexión, de manera especial, cuando ya hay "señales" que indican cómo la antropología está, por ejemplo, abriéndo- 
se a todo lo que supone la investigación genómica (vid. "Inferring patterns of folktale diffusion using genomic data". Proceedings of the National Academy of Sciences of United States of America, PNAS, vol. 114, n. ${ }^{\circ} 34$, Eugenio Bortolini, 9140-9145 , doi: 10.1073 / pnas.1614395114E <http://www.pnas.org/content/114/34/9140>), ya se están desarrollando proyectos que apuestan por poner de manifiesto la importancia del conocimiento antropológico para el estudio del cambio climático ("What knowledge societies can learn from fraging societies?", FP7-IDEAS-ERC, <http://icta.uab.at/etnoecologia/lek/>) o, incluso, el análisis antropológico se implica en el estudio de las nuevas tecnologías (a modo de ejemplo, vid. Mikael Johansson. "Towards an anthropology of nanoscience". Anthropology Today, vol. 19 n. ${ }^{\circ}$ 6, diciembre de 2003).

Asimismo, la propia comunidad científica de carácter experimental está reclamando, cada vez más, la participación de disciplinas como la antropología para abordar los problemas de cambio global; de esta manera, muchos de los paneles dedicados a clima, agua, etc., que ahora se han agrupado en el proyecto FUTURE EARTH (<http://www.futureearth.org/>), desarrollan diversos proyectos de carácter transversal que, una vez vistos sus objetivos, deberían ser, cuando menos, objeto de atención y estímulo sobre posibles líneas de investigación futuras para la antropología. En este sentido se cita una idea de cada uno de los programas con vistas a ver la interrelación de las propuestas con la antropología: Global Land Programme (GLP) (“[... ] how human activities on land are affecting feedbacks to the Earth System"); Global Mountain Biodiversity Assessment (GMBA) (" [...] in charge of the conservation and sustainable use of biodiversity in mountains regions"); Integrated History and Future of People on Earth (IHOPE) (" [... ] integrative frameworks to combine study human and Earth system history on behalf of our species' future”); oneHEALTH (" [... ] links between global environmental change and health for the planet and society”); Programme on Ecosystem Change and Society (PECS) ("[... relationships among natural capital, human wellbeing, livehoods, inequality and poverty"). En la actualidad, existen ejemplos de primer orden de cómo esa necesidad de aunar esfuerzos entre científicos de diversos ámbitos empieza a ser norma. En este sentido, una magnífica muestra de tal tarea puede ser el proyecto "La montagne et ses savoirs" (convocatoria PICSProjet International de Coopération Scientifique de Francia, 2013-2015), que agrupó a biológos, botánicos, lingüistas, antropólogos e historiadores de la re- 
ligión ( fuqahâ' y 'ulamâ') que tuvieron como objetivo conocer todo lo referido a los saberes tradicionales, usos, hablas y demás de la montaña en el Rif ( $v i d$. $<$ http://jbala.hypotheses.org/1978>).

\section{A modo de conclusión}

Una de las características de la antropología ha sido, y es, su propia reflexión crítica, la cual la ha hecho avanzar tanto en lo referido a sus propias argumentaciones teóricas como metodológicas. Este punto de vista también ha significado que la disciplina ha indagado en temas y problemas siempre amparada en el relativismo y en la defensa de los derechos humanos obviando los prejuicios de lo políticamente correcto. Teniendo presentes estos aspectos y con base en lo aquí expuesto, la antropología debería intentar acercarse a los grandes retos y desafíos actuales, al menos a los planteados en el seno de la UE. La valía intrínseca y añadida que el conocimiento antropológico tiene en sí mismo es un elemento que debería explotarse a fondo, pero ello conlleva, entre otras cosas, que posiblemente la disciplina debería hacer frente a nuevas formalizaciones y conceptualizaciones con el objetivo de no devenir una materia irrelevante.

Por todo ello, me pregunto si quizás no ha llegado el momento de revisar y volver a pensar algunas de las verdades antropológicas tradicionales que se han convertido en la seña identificativa de la disciplina, al menos en Cataluña. Pienso que si no se dan pasos en esa dirección, puede ser que la antropología pueda quedar como un «objeto exótico» en los proyectos del sistema de $\mathrm{I}+\mathrm{D}+\mathrm{I}$, tanto estatal como europeo, $y$, en definitiva, que la sociedad perciba la disciplina como un repertorio de descripciones de prácticas y símbolos cuya aportación sea irrelevante a la resolución de problemas.

De esta manera, y como punto final, estos aspectos me llevan a formular preguntas como las siguientes: ¿cómo incorporar y participar de los grandes desafíos actuales de la humanidad tanto en lo referido a la naturaleza ( «la era del Antropoceno») como a lo cultural (ciudades globales-megalópolis, migraciones, refugiados, exclusión...)?, ¿quizás la antropología del nuevo milenio esté obligada a centrarse en la resolución de problemas?, ¿̨cómo incorporar los nuevos instrumentos de análisis científico (ADN, análisis socioecológicos...) en la investigación antropológica de carácter cultural y social?, ¿quizás habría que recuperar la visión disciplinar más integradora planteada por Esteva Fabregat 
en el congreso de 1977? ¿̨habría que ir hacia una profesionalización más allá de la academia, de los museos y de las entidades de la sociedad civil, y de esta guisa, sería posible pensar en otros instrumentos corporativos que pudiesen impulsar socialmente la antropología, tales como un colegio profesional? 